\title{
The synergy factor: a statistic to measure interactions in complex
} diseases

\author{
Mario Cortina-Borja ${ }^{1}$, A David Smith ${ }^{2}$, Onofre Combarros*3,4 and \\ Donald J Lehmann²
}

\begin{abstract}
Address: ${ }^{1}$ Centre for Paediatric Epidemiology and Biostatistics, Institute of Child Health, University College London, 30 Guilford Street, London, WC1N 1EH, UK, 2Oxford Project to Investigate Memory and Ageing (OPTIMA), Department of Physiology, Anatomy and Genetics, South Parks Road, Oxford, OX1 3QX, UK, ${ }^{3}$ Neurology Service and Centro de Investigación Biomédica en Red sobre Enfermedades Neurodegenerativas (CIBERNED), Sevilla, Spain and ${ }^{4}$ Marqués de Valdecilla University Hospital (University of Cantabria), 39008 Santander, Spain

Email: Mario Cortina-Borja - m.cortina@ich.ucl.ac.uk; A David Smith - david.smith@pharm.ox.ac.uk; Onofre Combarros* - combarro@unican.es; Donald J Lehmann - donald.lehmann@pharm.ox.ac.uk

* Corresponding author
\end{abstract}

Published: 15 June 2009

BMC Research Notes 2009, 2:105 doi:10.1186/1756-0500-2-105
Received: 12 December 2008

Accepted: 15 June 2009

This article is available from: http://www.biomedcentral.com/I756-0500/2/105

(c) 2009 Combarros et al; licensee BioMed Central Ltd.

This is an Open Access article distributed under the terms of the Creative Commons Attribution License (http://creativecommons.org/licenses/by/2.0), which permits unrestricted use, distribution, and reproduction in any medium, provided the original work is properly cited.

\begin{abstract}
Background: One challenge in understanding complex diseases lies in revealing the interactions between susceptibility factors, such as genetic polymorphisms and environmental exposures. There is thus a need to examine such interactions explicitly. A corollary is the need for an accessible method of measuring both the size and the significance of interactions, which can be used by nonstatisticians and with summarised, e.g. published data. The lack of such a readily available method has contributed to confusion in the field.

Findings: The synergy factor (SF) allows assessment of binary interactions in case-control studies. In this paper we describe its properties and its novel characteristics, e.g. in calculating the power to detect a synergistic effect and in its application to meta-analyses. We illustrate these functions with real examples in Alzheimer's disease, e.g. a meta-analysis of the potential interaction between a BACEI polymorphism and APOE4: $S F=2.5,95 \%$ confidence interval: I.5-4.2; $p=0.000 \mathrm{I}$.

Conclusion: Synergy factors are easy to use and clear to interpret. Calculations may be performed through the Excel programmes provided within this article. Unlike logistic regression analysis, the method can be applied to datasets of any size, however small. It can be applied to primary or summarised data, e.g. published data. It can be used with any type of susceptibility factor, provided the data are dichotomised. Novel features include power estimation and meta-analysis.
\end{abstract}

\section{Background \\ The need}

The remarkable progress made in the understanding of single-cause diseases has not yet been matched in the study of complex conditions. One problem is that susceptibility factors, e.g. genetic and environmental, all contrib- ute risk that is to varying extents contingent on the presence of other factors [1-4]. Complex diseases cannot therefore be simply seen as due to the accumulation of many small independent effects. Rather, their very complexity lies in the interactions between contingent effects. Important effects may thus be missed if only single factors 
are independently examined (Discussion). The study of interactions between risk factors is thus central to the study of complex diseases.

Yet, unravelling interactions has proved confusing (Discussion). There is a need for a readily accessible method of measuring their strength, available to non-statisticians and applicable to summarised data and to datasets of any size. Methods are also needed to calculate the power to detect an interaction and to perform meta-analyses of interactions from published data; these two functions have not so far been readily available. There is a particular need for an accessible method for referees; untested claims of synergy are regularly published. Here we present a statistic, the synergy factor $(S F)$, derived from logistic regression models, which aims to address these needs.

\section{Modelling interactions in case-control studies}

This paper is about statistical interactions; thus, drawing inferences about biological causality is beyond its scope. In general, a statistical interaction arises "when the effect of one explanatory variable depends on the particular level or value of another explanatory variable" [5]. Interactions may correspond to deviations from additive or multiplicative models for the joint effects of two risk factors. This has been thoroughly explored by Berrington de González and Cox $[6,7]$, with two procedures, one for each model.

Some epidemiologists, e.g. Rothman and Greenland [8], argue that assessment of interaction should be based on additive rate or risk models. These models are the norm in cohort studies. However, to assess interaction as departure from additive risks in case-control studies, three surrogate measurements of interaction based on the parameters of logistic regression models have been proposed $[9,10]$ : the relative excess risk due to interaction, the attributable proportion due to interaction and the synergy index. Skrondal has shown [11] that only the synergy index may be validly used for this purpose and only after fitting a linear odds model.

In case-control studies, the parameter which is both estimable and interpretable as a relative risk is the odds ratio $(O R)$ [11]. In such studies, the predicted joint effect of two genetic or other factors may be defined as the product of the effects of each factor alone. We therefore propose a single statistic, the synergy factor $(S F)$, which depends on a multiplicative definition of the null hypothesis.

\section{Methods}

A full description of the methodology for significance tests based on the $S F$ appears in Additional file 1. We show there that $\ln (S F)$ is equivalent to the interaction term defined by two binary factors in a logistic regression model. We test the hypothesis of no interaction, using a Normal approximation for the statistic $\ln (S F) /$ stderr $(\ln (S F))$, where the standard error of $\ln (S F)$ is easily obtained via the delta method [12]. This approximation is adequate even for relatively small sample sizes. We discuss a modification of the $S F$ to cope with empty cells and propose two bootstrap approximations and a Bayesian inferential procedure that can be used as alternatives to the Normal approximation. We also propose methodology to calculate the power of significance tests and to perform meta-analyses based on the SF.

\section{Results}

\section{The synergy factor (SF)}

Let us assume we wish to estimate from a case-control study whether there is an interaction between any two (binary) factors, $x_{1}$ and $x_{2}$, in the risk of a certain (binary) condition. Taking subjects with neither factor as reference, we first estimate the ORs for factor $x_{1}$ alone $\left(O R_{1}\right)$, factor $x_{2}$ alone $\left(O R_{2}\right)$ and both factors combined $\left(O R_{12}\right)$. The $S F$ is then defined as: $S F=O R_{12} /\left(O R_{1} \times O R_{2}\right)$ and is the ratio of the observed $O R$ for both factors combined, to the predicted $O R$ assuming independent effects of each factor. Susceptibility factors may be associated with increased or reduced risk, i.e. risk or protective factors, respectively (we make no assumptions about causality). In either case, interactions may be positive (synergy) or negative (antagonism). Thus, if $S F>1(<1)$, then there is a positive (negative) interaction between two risk factors. The opposite applies to protective factors.

To obtain the statistical significance of $S F$, construct a $4 \times$ 2 table of the numbers of cases and controls in each of the 4 possible combinations of the two factors (e.g. Table 1). Then if $n_{1}, n_{2} \ldots . n_{8}$ are the values of the 8 cells, application of the delta method [12] yields an asymptotic normal approximation to the standard error of $\ln (S F)$ as: $\operatorname{stderr}(\ln (S F))=\sqrt{1 / n_{1}+1 / n_{2}+\cdots+1 / n_{8}}$. Since the null value is 0 , the statistic $Z=\ln (S F) / \operatorname{stderr}(\ln (S F))$ has asymptotically a standard normal distribution under the null hypothesis of no interaction.

Table I: Odds ratios of Alzheimer's disease, taking subjects with the BACEI rs638405 C allele and without APOE4 as reference

\begin{tabular}{lllll}
\hline BACEI & APOE4 & Controls & Cases & OR \\
\hline C+ & - & 125 & 80 & Reference \\
GG & - & 80 & 38 & 0.742 \\
C+ & + & 48 & 74 & 2.409 \\
GG & + & 19 & 60 & 4.934 \\
\hline Totals & & 272 & 252 &
\end{tabular}

Data from Nowotny et al 200I [13].APOE4 = the $\varepsilon 4$ allele of apolipoprotein E; BACEI = the $\beta$-site APP-cleaving enzyme; $C+$ and GG refer to BACEI exon 5 C/G (rs638405) genotypes; APOE4+ and $B A C E I C+$ pool homozygotes and heterozygotes of the respective alleles; $O R=$ odds ratio 


\section{Synergy between risk factors}

Let us take the potential interaction in risk of Alzheimer's disease (AD) between the $\varepsilon 4$ allele of apolipoprotein $\mathrm{E}$ (APOE4) and the GG genotype of the $\mathrm{C} / \mathrm{G}$ polymorphism (rs638405) in exon 5 of the $\beta$-site APP-cleaving enzyme (BACE1) [13] (Table 1). Taking subjects with neither BACE1 GG nor APOE4 as reference, the OR for BACE1 GG alone was 0.742 and that for APOE4 alone was 2.409 . That gave a predicted $O R$ of $1.788(=0.742 \times 2.409)$ for the combination, compared with an observed $O R$ of 4.934. Hence: $S F=2.76(=4.934 / 1.788), 95 \%$ confidence interval (CI): 1.25-6.09, $\ln (S F)=1.015, \operatorname{stderr}(\ln (S F))=$ $0.404, Z=2.25$ and $p=0.012$. Thus the null hypothesis of no interaction was rejected and significant synergy was found. The observed joint effect of the two variants was nearly three times greater than the predicted joint effect.

Using the data of Table 1, we also calculated a bootstrap approximation (see Methods) to the null distribution of $\ln (S F)$, based on 10,000 simulated samples. This approximation does not depend on an asymptotic argument and gave $p=0.006$. Figure 1 shows both the normal and the

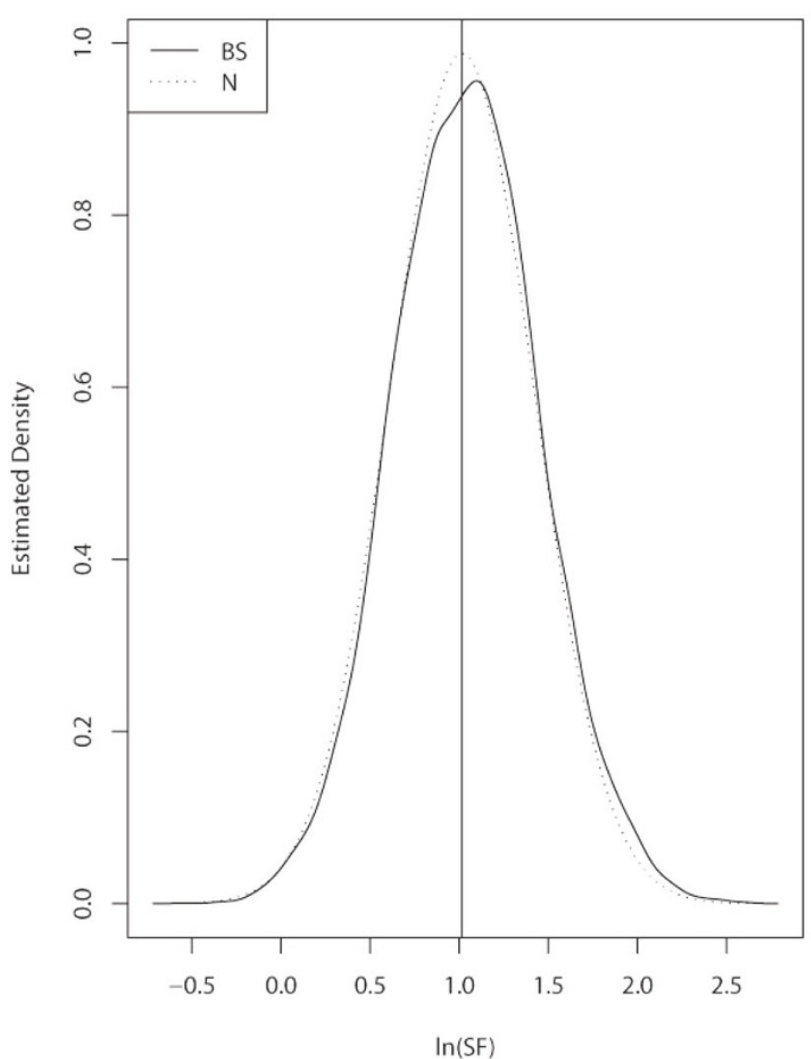

bootstrap approximations. The left-hand plot shows the density estimate for the bootstrap approximation and the normal density, with mean and standard deviation given by the observed $\ln (S F)$ and the standard error: stderr $(\ln (S F))=\sqrt{1 / n_{1}+1 / n_{2}+\cdots+1 / n_{8}}$; the right-hand plot compares the sample quantiles of the bootstrap values of $\ln (S F)$ with those of a standard normal distribution, shown as a straight line. Both graphs confirm the adequacy of the normal approximation, which we also tested formally using the Kolmogorov-Smirnov test $(p=0.66)$.

The above example is of synergy between risk factors. Examples of antagonism and of protective factors are given in Additional file 2. SF calculations may be performed using the Excel programme in Additional file 3; an $\mathrm{R}$ function is available (from MCB) to compute the bootstrap approximation.

\section{Power}

Figure 2 shows power functions for different total sample sizes based on the control exposure frequencies presented

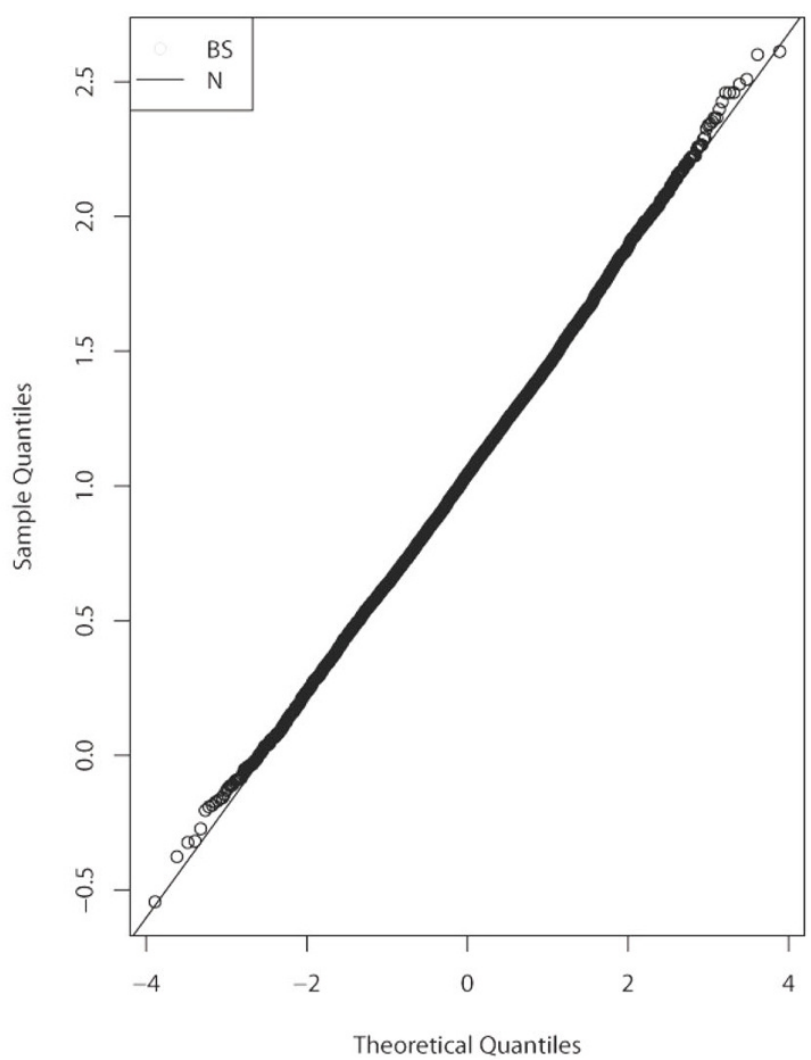

Figure I

Normal (N) and bootstrap (BS) approximations to the null distribution of In(SF). These are based on the data in Table I. On the right is the normal Quantile-Quantile plot for the values obtained by the bootstrap procedure. 
in Table 1, i.e. $36 \%$ and $25 \%$ for BACE1 GG and APOE4, respectively. The total sample size of that study [13] was 524 , and we also calculated the power functions corresponding to total sample sizes of 200, 1000, 2000, and 4000. When the SF equals 1, the power is 0.05 , which is the significance level used. In this example, 488 cases and 488 controls will be needed to have $80 \%$ power to detect an SF of 2. Power calculations may be performed on $\mathrm{R}$, using a function available from MCB.

\section{Meta-analyses}

Table 2 shows the data from 4 studies of the interaction between APOE4 and the BACE1 exon 5 GG genotype in the risk of AD [13-16]. These 4 studies are the only studies in Caucasians currently providing data to examine this interaction. Taking subjects with neither BACE1 GG nor $A P O E 4$ as reference, the pooled SF obtained using the random effects model [17] was 2.51, with 95\% CI: 1.50-4.19 and $p=0.0001$. The heterogeneity statistic based on three degrees of freedom was $1.88(p=0.76)$; the estimated random effects variance was 0 . The results appear in Figure 3.
Meta-analyses may be performed using the Excel programme in Additional file 4.

\section{Discussion}

\section{The need}

The real examples in Tables 1 and 2 and Table S1-S3 [Additional file 2] show the dangers of neglecting interactions. In all these examples, the effects of one or both variants were completely masked by the interacting factor. For instance, in the meta-analysis of four BACE1 studies (Table 2 and Figure 3), the effect of the BACE1 exon 5 GG was hidden in the absence of APOE4 [pooled $O R=0.8$ (95\% CI: $0.6-1.1 ; p=0.17$ ), random effects model [17]], but revealed in its presence [1.9 (1.3-2.9; 0.0015)]. Tables S1-S3 [Additional file 2] give further examples of such masking.

There is a common view that interactions, e.g. between genes (epistasis), should only be examined between risk factors that have already shown a significant main effect. But in many cases, such as most of the above, the associa-

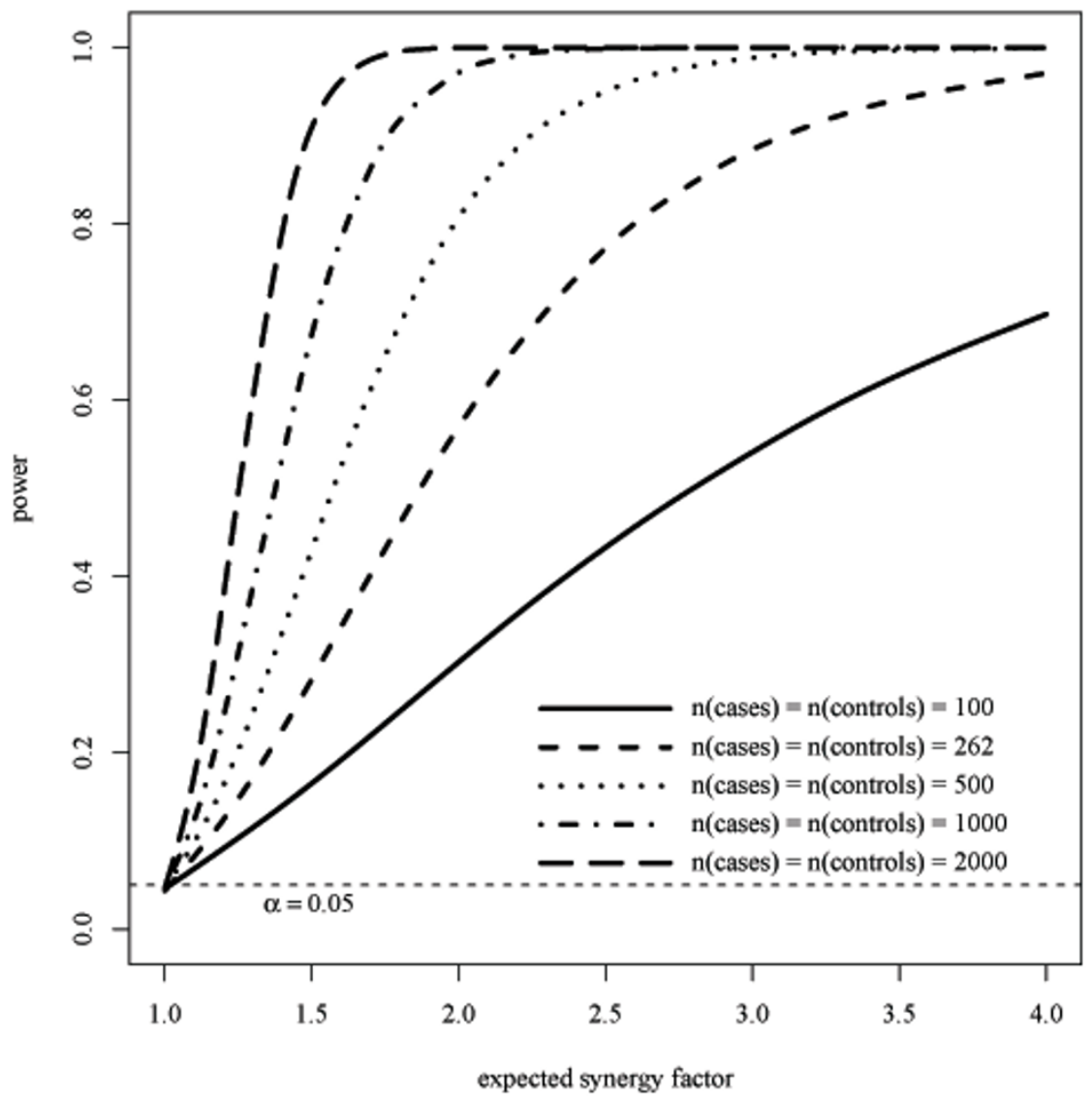

Figure 2

Power curves for various sample sizes based on the control exposure frequencies in Table I. The example with 262 cases and 262 controls is equivalent to that of Table I with 252 cases and 272 controls. 


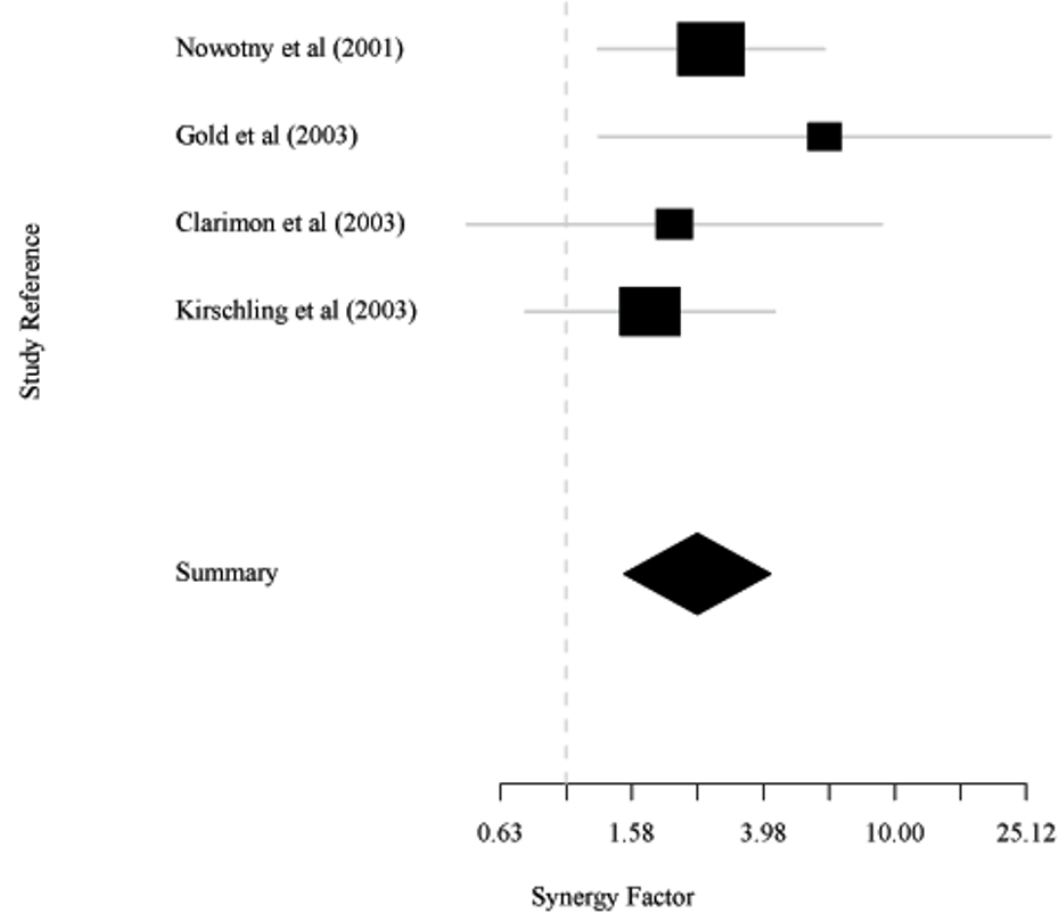

Figure 3

Meta-analysis of the interaction between BACEI GG and APOE4. This is based on a random effects model [I7].

tion would be missed by the traditional single-factor approach [1-3]. Indeed, this was so in most of the examples of significant epistasis uncovered in our recent survey of sporadic AD [18]. Out of 36 such examples, 34 with SFs $\geq 2$, the main effects of the gene variants other than APOE4 were generally very weak. The ORs were $\leq 1.2$ in 20 out of 36 cases and were only significant in 5 cases. Thus, preliminary screening for main effects will miss many, possibly most cases of epistasis.

On the other hand, synergy can be too easily claimed. A common misconception is that a high combined OR necessarily implies synergy. A single $O R$ by itself says nothing about synergy; it is the relation between the three relevant
ORs that matters. For instance, let us assume that two risk factors are associated with ORs of 3 and 5 alone and of 15 when combined. Although the combined value is impressive, there is no synergy: $S F=15 /(3 \times 5)=1$. Claims of synergy are frequently published on the basis of such invalid evidence. Indeed, we have noted at least 20 claims of interactions, in the field of AD genetics alone, that were published in leading journals in recent years, but which may be clearly refuted by $S F$ analysis. There is thus a need for a readily accessible method of testing such claims.

\section{Limitations of the SF method}

We suggest that $S F$ analysis, being based on logistic regression analysis, is best used for assessing binary interactions

Table 2: Data for an SF meta-analysis of the interaction between BACEI rs638405 GG and APOE4

\begin{tabular}{|c|c|c|c|c|c|c|c|c|}
\hline \multirow[t]{2}{*}{ Study } & \multicolumn{2}{|c|}{$\begin{array}{l}\text { APOE4-positive, } \\
\text { BACEI GG }\end{array}$} & \multicolumn{2}{|c|}{$\begin{array}{l}\text { APOE4-positive, } \\
\text { BACEI C+ }\end{array}$} & \multicolumn{2}{|c|}{$\begin{array}{l}\text { APOE4-negative, } \\
\text { BACEI GG }\end{array}$} & \multicolumn{2}{|c|}{$\begin{array}{l}\text { APOE4-negative, } \\
\text { BACEI C+ }\end{array}$} \\
\hline & Controls & Cases & Controls & Cases & Controls & Cases & Controls & Cases \\
\hline Nowotny et al 200I [13] & 19 & 60 & 48 & 74 & 80 & 38 & 125 & 80 \\
\hline Gold et al 2003 [15] & 3 & 14 & 16 & 16 & 41 & 16 & 90 & 46 \\
\hline Clarimon et al $2003[14]$ & 4 & 40 & 10 & 40 & 21 & 18 & 52 & 38 \\
\hline Kirschling et al 2003 [16] & 22 & 48 & 40 & 62 & 63 & 22 & 112 & 50 \\
\hline
\end{tabular}

APOE4 = the $\varepsilon 4$ allele of apolipoprotein $\mathrm{E} ; \mathrm{BACEI}=$ the $\beta$-site APP-cleaving enzyme; $\mathrm{GG}$ and $\mathrm{C}+$ refer to BACEI exon $5 \mathrm{C} / \mathrm{G}$ (rs638405) genotypes; APOE4-positive and BACEI C+ pool homozygotes and heterozygotes of the respective alleles 
[2]. Various methods have been devised to examine higher order interactions $[19,20]$. However, some have only limited value for purposes of interpretation. Moreover, nearly all case-control sample-sets currently used for association studies lack the power for the proper study of higher order interactions [18]. Where a third interacting factor is suspected and a sufficiently large dataset is available, $S F$ analysis may be performed twice, after stratification by the third factor, e.g. gender.

Where the relevant data are available, logistic regression analysis is the appropriate method for adjusting for covariates, while $S F$ analysis should be the preferred method for stratification by covariates. Stratification can produce very small subsets, even of zero, which logistic regression analysis cannot handle. In contrast, $S F$ analysis produces a realistic $p$ value in each subgroup, if one adds 0.5 to each cell in any $4 \times 2$ table with at least one zero cell $[21,22]$.

\section{Advantages of the SF method}

SF analysis is simple to perform, through the Excel programmes in Additional files 3 and 4. It is a matter of a few minutes to perform the analysis, e.g. to check a claim of synergy in a published paper. The value of the method may be seen in the study of Combarros et al 2008 [18], in which $S F$ analysis was used to examine each of the 89 studies of interactions cited in that review. The method measures both the size and significance of a binary interaction, using either primary or summarised data. Unlike logistic regression analysis, it can be applied to datasets of any size, however small, even with zero cells (above). The method can be used with all types of susceptibility factors, both risk and protective, for instance, age, gender, diet, medication or genetic polymorphisms, provided the data are dichotomised, e.g. age \pm 75 years. It can be applied both to synergistic and to antagonistic interactions. Novel features include power estimation (through an R function available from MCB) and meta-analysis, an increasingly important application (through the Excel programme in Additional file 4). Neither function has been readily available before.

\section{Competing interests}

The authors declare that they have no competing interests.

\section{Authors' contributions}

MCB and DJL conceived the method. MCB devised the various statistical applications and worked out the necessary proofs. ADS advised on applications, e.g. in AD genetics. OC and DJL selected real examples from the $\mathrm{AD}$ literature and tested the method on those examples. MCB and DJL wrote the initial drafts of the manuscript and all authors read those drafts and contributed to the revisions. All authors approved the final manuscript. OC submitted the article.

\section{Additional material}

\section{Additional file 1}

Methods. text +8 references.

Click here for file

[http://www.biomedcentral.com/content/supplementary/1756-

0500-2-105-S1.doc]

\section{Additional file 2}

Examples of various types of interactions. text +3 tables +3 references. Click here for file

[http://www.biomedcentral.com/content/supplementary/1756-

0500-2-105-S2.doc]

\section{Additional file 3}

Cortina-Borja 2009, SF calculator. Excel programme.

Click here for file

[http://www.biomedcentral.com/content/supplementary/1756-

0500-2-105-S3.xls]

\section{Additional file 4}

Cortina-Borja 2009, SF calculator, meta-analysis. Excel programme with macro.

Click here for file

[http://www.biomedcentral.com/content/supplementary/17560500-2-105-S4.xls]

\section{Acknowledgements}

We are most grateful to $\mathrm{Dr}$ Jonathan Marchini for his detailed reading of a previous version of the manuscript and to Dr Kirsty Little for her advice on implementing the procedure for doing meta-analyses in Excel. Most of this work was undertaken at GOSH/UCL Institute of Child Health, which received a proportion of funding from the Department of Health's NIHR Biomedical Research Centres funding scheme. The Centre for Paediatric Epidemiology and Biostatistics also benefits from funding support from the Medical Research Council in its capacity as the MRC Centre of Epidemiology for Child Health (G0400546). OPTIMA is supported by major grants from the Charles Wolfson Charitable Trust and Merck Inc. and is a centre in the Alzheimer's Research Trust Network.

\section{References}

I. Culverhouse R, Suarez BK, Lin J, Reich T: A perspective on epistasis: limits of models displaying no main effect. Am J Hum Genet 2002, 70:46I-47I.

2. Moore JH, Williams SM: New strategies for identifying genegene interactions in hypertension. Ann Med 2002, 34:88-95.

3. Moore $\mathrm{JH}$ : The ubiquitous nature of epistasis in determining susceptibility to common human diseases. Hum Hered 2003, 56:73-82.

4. Pembrey M: The Avon Longitudinal Study of Parents and Children (ALSPAC): a resource for genetic epidemiology. The ALSPAC Study Team. Eur J Endocrinol 2004, 15 I:UI25-UI 29.

5. Fitzmaurice $G$ : The meaning and interpretation of interaction. Nutrition 2000, 16:3|3-314.

6. Berrington de González A, Cox DR: Additive and multiplicative models for the joint effect of two risk factors. Biostatistics 2005, 6:1-9.

7. Berrington de González A, Cox DR: Interpretation of interaction: a review. Ann Appl Stat 2007, I:37|-385.

8. Rothman KJ, Greenland S: Modern Epidemiology. 2nd edition. Philadelphia: Lippincott-Raven; 1998.

9. Rothman KJ: Synergy and antagonism in cause-effect relationships. Am J Epidemiol 1974, 99:385-388. 
10. Rothman KJ: The estimation of synergy or antagonism. Am J Epidemiol 1976, 103:506-5II.

II. Skrondal A: Interaction as departure from additivity in casecontrol studies: a cautionary note. Am J Epidemiol 2003, I 58:25I-258.

12. Tanner M: Tools for statistical inference. Berlin: Springer-Verlag; 1990.

13. Nowotny P, Kwon JM, Chakraverty S, Nowotny V, Morris JC, Goate AM: Association studies using novel polymorphisms in BACEI and BACE2. Neuroreport 200I, I 2: 1799-1802.

14. Clarimón J, Bertranpetit J, Calafell F, Boada M, Tàrraga L, Comas D: Association study between Alzheimer's disease and genes involved in $A \beta$ biosynthesis, aggregation and degradation: suggestive results with BACEI. J Neurol 2003, 250:956-96I.

15. Gold G, Blouin JL, Herrmann FR, Michon A, Mulligan R, Duriaux Saïl G, Bouras C, Giannakopoulos P, Antonarakis SE: Specific BACE I genotypes provide additional risk for late-onset Alzheimer disease in APOE $\& 4$ carriers. Am J Med Genet 2003, I I 9B:44-47.

16. Kirschling CM, Kölsch H, Frahnert C, Rao ML, Maier W, Heun R: Polymorphism in the BACE gene influences the risk for Alzheimer's disease. Neuroreport 2003, 14:1243-1246.

17. Der Simonian R, Laird N: Meta-analysis in clinical trials. Control Clin Trials 1986, 7:177-188.

18. Combarros O, Cortina-Borja M, Smith AD, Lehmann DJ: Epistasis in sporadic Alzheimer's disease. Neurobiol Aging 2008 in press.

19. Musani SK, Shriner D, Liu N, Feng R, Coffey CS, Yi N, Tiwari HK, Allison DB: Detection of gene $\times$ gene interactions in genomewide association studies of human population data. Hum Hered 2007, 63:67-84.

20. Thornton-Wells TA, Moore JH, Haines JL: Genetics, statistics and human disease: analytical retooling for complexity. Trends Genet 2004, 20:640-647.

21. Anscombe Fj: On estimating binomial response relations. Biometrika 1956, 43:46I-464.

22. Breslow N: Odds ratio estimators when the data are sparse. Biometrika 1981, 68:73-84.

Publish with Bio Med Central and every scientist can read your work free of charge

"BioMed Central will be the most significant development for disseminating the results of biomedical research in our lifetime. "

Sir Paul Nurse, Cancer Research UK

Your research papers will be:

- available free of charge to the entire biomedical community

- peer reviewed and published immediately upon acceptance

- cited in PubMed and archived on PubMed Central

- yours - you keep the copyright 